\title{
Multiplexed affinity measurements of extracellular vesicles binding kinetics
}

\author{
Authors: \\ Elisa CHIOdI \\ Department of Electrical Engineering \\ Boston University, Boston (MA) \\ George DaAboul \\ Nanoview Biosciences \\ Boston (MA) \\ Allison M. MARN \\ Department of Electrical Engineering \\ Boston University, Boston (MA) \\ M. Selim ÜNLÜ \\ Department of Electrical Engineering \\ Boston University, Boston (MA)
}




\begin{abstract}
Extracellular vesicles (EVs) have attracted significant attention as impactful diagnostic biomarkers, since their properties are closely related to specific clinical conditions. However, designing experiments that involve EVs phenotyping is usually highly challenging and time-consuming, due to laborious optimization steps that require very long or even overnight incubation durations. In this work, we demonstrate label-free, real-time detection and phenotyping of extracellular vesicles binding to a multiplexed surface. With the ability of label-free kinetic binding measurements using the Interferometric Reflectance Imaging Sensor (IRIS) in a microfluidic chamber, we successfully optimize the capture reaction by tuning various assay conditions (incubation time, flow conditions, surface probe density and specificity). A single (less than 1 hour) experiment allows for characterization of binding affinities of the EVs to multiplexed probes. We demonstrate kinetic characterization of 18 different probe conditions, namely three different antobodies, each spotted at six different concentrations, simultaneously. The affinity characterization is then analyzed through a model which considers the complexity of multivalent binding of large structures to a carpet of probes, and therefore introduces a combination of fast and slow association and dissociation parameters. Additionally, our results confirm higher affinity of EVs to aCD81 with respect to aCD9 and aCD63. Single-vesicle imaging measurements corroborate our findings, as well as confirming the EVs nature of the captured particles through fluorescence staining of the EVs membrane and cargo.
\end{abstract}

Keywords - Label-free biosensor, extracellular vesicles (EVs), EVs detection, Microarray, Interferometric imaging

\section{Introduction}

In the past few years, the interest in Extracellular Vesicles (EVs) as theranostic tools has significantly increased [1]. These biological particles constitute a very heterogenous population in the human body, both for origin and size. They range from vesicles of endosomic origin (small EVs, or exosomes, 50$150 \mathrm{~nm})$ to microvescicles $(500 \mathrm{~nm}-1 \mu \mathrm{m})$ released from the plasma membrane [2]. The heterogeneity of these biological nanoparticles can sometimes pose a challenge in terms of purification and phenotyping.

When they were first discovered, EVs were considered to be a cellular discard. They soon revealed their potential, proving to be a valuable asset in the field of biomarker discovery and therapy design. By carrying pieces of information in the form of RNA fragments and biomarkers, EVs act as a 'journal' of each individual's health conditions and make a crucial contribution to the intracellular communication.

One of the main challenges of research on
EVs is the time-consuming nature of experiments including laborious purification processes. Moreover, in order to get accurate results, maximizing binding efficiency of the vesicles to their specific probe is a necessity; for end-point measurements, that implies ensuring the saturation of the capture reaction, which often results in an unnecessarily long incubation time. A typical phenotyping experiment involves a $12 \mathrm{~h}$ - or overnight incubation of the sensor chips with the EVs sample, thus causing a significant delay in data acquisition and interpretation. In order to exploit the potential utilization of EV biomarkers, faster and high-throughput analysis methods and tools are needed.

To address this need, a variety of real-time detection methods for extracellular vesicles have been studied [3, 4]. For example, it has been demonstrated that a good technique to monitor the behavior of EVs in real time is to label them with either a fluorescent probe (Real-Time Fluorescence Microscopy Single Particle Tracking (SPT) [5]) or a gold nanoparticle $[6,7,8]$. Labeling offers great sensitivity and tagging with fluorescent/gold- 
labeled anti-tetraspanins ensures the specificity of the tracked particle. However, it is an indirect method that can be prone to artifacts such as non-specific binding and photo-instability of the fluorescent molecules, or alteration of the binding affinity due to Au labels with larger mass than the EV itself.

Another established method for the real-time detection of extracellular vesicles is Surface Plasmon Resonance Imaging (SPRi) $[9,10,11,12]$. This technique succeeds in monitoring the labelfree binding of EVs to different probes simultaneously. However, as in the case of all evanescentwave based sensors, SPR cannot distinguish surface binding from local changes in solution refractive index and it is prone to environmental factors (temperature, vibrations, $\mathrm{pH}$ variations). Since EVs are normally purified from plasma or cell culture media, the typical target solutions are highly heterogenous. Therefore, the large variations in refractive index further exacerbate the background noise in SPR measurements.

In this work, we developed a whole new application for kinetic measurements on Interferometric Reflectance Imaging Sensor by tailoring it to the multiplexed phenotyping of EVs. By quantifying the thickness accumulated on the surface, and by removing any unwanted background effects through differential measurements, we were able to precisely evaluate the dynamic accumulation of EVs. Through the use of low-magnification optics, we combined real-time detection and multiplexing, simultaneously measuring the real-time capture of EVs on three distinct antibody surfaces (aCD9, aCD63, aCD81), each immobilized at six different concentrations. This way, we could efficiently optimize the probe density, probe specificity, incubation time, and flow velocity, in a single experiment. The acquisition time was maintained below one hour per experiment, therefore yielding a reliable, quantitative, easy optimization method for EVs characterization.

The kinetic results obtained with the IRIS were corroborated by single-particle measurements, both label-free and labeled, that were performed on the ExoView ${ }^{\mathrm{TM}}$ system (Section 3.1).

\section{Materials and Methods}

\subsection{EVs culture and purification}

HEK293T cell line was cultured using Dulbecco's modified Eagle's medium (DMEM) (Thermo Scientific) supplemented with $10 \%(\mathrm{v} / \mathrm{v})$ of FBS (Gibco, Invitrogen). Upon confluence, the cells were washed twice with dPBS (Gibco, Invitrogen) and incubated in DMEM supplemented with 10\% (v/v) of exosome depleted FBS (Gibco, Invitrogen) for $48 \mathrm{~h}$. Conditioned media was then collected and centrifuged at $2500 \mathrm{x} \mathrm{g}$ for 15 mins at room temperature to remove cellular debris. The supernatant was transferred to a new tube and was centrifuged at $2500 \mathrm{x}$ g for $15 \mathrm{mins}$ at room temperature. Finally, the supernatant was aliquoted and stored at $-80^{\circ} \mathrm{C}$ until usage.

EVs were concentrated through ultracentrifugation to allow binding measurements on the IRIS system. To concentrate the EVs $60 \mathrm{~mL}$ of conditioned media was pelleted by ultracentrifugation at $110^{\prime} 000 \mathrm{x}$ g for 16 hours at $4^{\circ} \mathrm{C}$ in S50A Rotor (Thermo Scientific). The pellet was resuspended into $60 \mathrm{~mL}$ of $1 \mathrm{X}$ PBS and pelleted again at 110,000 $\mathrm{x} g$ for 6 hours at $4^{\circ} \mathrm{C}$. The pellet was then resuspended in $.5 \mathrm{~mL}$ of PBS.

Prior to the IRIS experiment, the EVs sample was centrifuged at $60^{\prime} 000 \mathrm{rpm}$ for 10 minutes to eliminate large aggregates. The surnatant was then diluted (2X) to be used for the experiment, while the pellet was discarded.

\subsection{The IRIS platform}

The Interferometric Reflectance Imaging Sensor (IRIS) (Figure S1) has been extensively described in many publications [13]. Briefly, a silicon chip with a layer of thermally grown silicon oxide $\left(\mathrm{SiO}_{2}\right)$ on top is used as a substrate, where biomolecules are immobilized through an active surface chemistry (described in Section 2.5).

The $\mathrm{Si} / \mathrm{SiO}_{2}$ substrate is illuminated from the top through a microfluidic chamber, and it acts as a common path interferometer, where light reflecting from top of the sensor surface interferes with 
the reference reflection at the oxide-Si interface. Accumulation of biological mass on the sensor surface increases the effective thickness from the top surface to the reference Si surface and alters the reflectance. In IRIS measurements, the molecular weight of the analyte can vary from small molecules [14] to macroparticles such as viruses [15]. The reflectance coming from the surface assumes the form:

$$
R=\left|r^{2}\right|=\frac{r_{1}^{2}+r_{2}^{2}+2 r_{1} r_{2} \cos 2 \phi}{1+r_{1}^{2} r_{2}^{2}+2 r_{1} r_{2} \cos 2 \phi}
$$

Where $\phi=\frac{2 \pi d}{\lambda} n_{\mathrm{SiO}_{2}}$.

The oxide thickness is engineered to provide maximum constructive interference in response to biomass accumulation, therefore resulting in an increase in signal from the active probe spots. By generating a lookup table [16], it is possible to convert this signal difference to mass per unit area, therefore allowing for quantitative surface density measurements. The $50-\mu \mathrm{m}$ thick microfluidic chamber allows for high flow rates and reduction of mass transport effect.

\subsection{The ExoView ${ }^{\mathrm{TM}}$ system}

The single particle characterization experiments of the EVs samples were carried out on the ExoView ${ }^{\mathrm{TM}}$ system by NanoView, BioSciences.

\subsection{Simulations, data acquisition and analysis}

Reflectance simulations providing the change in reflectance due to the accumulation of EVs on the sensor surface were performed in MATLAB.

The real-time images were acquired through Micro-Manager software. Between subsequent frames, a fixed interval $60 \mathrm{~ms}$ was always maintained, and 100 frames were averaged to obtain one image. Therefore, all data points are 6s-spaced. Taking into account the results of the reflectance simulations, the videos could be converted to mass per unit area by applying a look-up table to the images, through a custom MATLAB code that uses the bare silicon region as a normalization reference. The images were then analyzed in ImageJ. A donut-shaped region of film around each spot was used as a background, and the differential mass density data thus obtained were plotted and fitted in MATLAB. For the IgG1 control experiment, a bivalent fitting model was applied. On the other hand, to fit the binding curves of exosomes, a more complex model was utilized, where a combination of fast and slow association and dissociation rates are considered [17], as further detailed in Section 4.2 .

\subsection{Surface chemistry and chip spot- ting}

Prior to probe immobilization, chips were coated with MCP-2 polymer (Lucidant). This reactive polymer is based on DMA-NAS-MAPS chemistry and has been extensively described in the literature [18].

To fabricate chips with different antibody density on the surface, the tetraspanin specific antibody was mixed with Mouse IgG2a. The total concentration of the spotted antibody was maintained at $3 \mathrm{mg} / \mathrm{mL}$, however, the ratio of tetraspanin specific antibody (CD81, CD63, and CD9) and Mouse IgG2a was varied. The antibody solutions were spotted onto the polymer coated chips using S12 sciFLEXARRAYER from ScienIon. The antibodies were allowed to immobilize on the surface for four hours in a humid chamber. Then the chips were washed is $1 \mathrm{X}$ PBST followed by a rinse in Millipore water and carefully dried under nitrogen stream.

\subsection{Antibodies and other reagents}

Antibodies used for making the chips and antibody labeling are anti-CD81 (JS-81), anti-CD63 (H5C6), anti-CD9 (HI9a), Mouse IgG1k, and Mouse IgG2a provided by NanoView Biosciences. 


\section{Results}

\subsection{EVs characterization by Single Particle Interferometric Imaging}

In order to satisfy the Minimal Information for Studies on Extracellular Vesicles (MISEV 2018 [19]) requirements, we characterized the studied EVs for size, protein content and surface properties. All the measurements were carried out on the ExoView system. To characterize the vesicles for their size, an ultra-centrifugated (UC) HEK EVs sample was diluted 1:100 and incubated on an SP-IRIS chip in order to capture EVs on three different antibody spots: CD9, CD63 and CD81. Label-free images of the capture spots were acquired on the ExoView system and counting and sizing of the particles was performed (Figure 1a). The size distribution is coherent with what expected for small EVs. A label-free image of the vesicles is shown in Figure 1b. Subsequently, the same chip was also stained with fluorescent antibodies for CD9, CD81 and CD63, and co-localization was determined (Figure $2 \mathrm{a}$ and $2 \mathrm{~b}$ ). This allowed to characterize the EVs in terms of their surface receptors. Co-localization has been determined to be high $(>50 \%)$ for all the antibodies.

Moreover, to analyze the EVs internal cargo, we stained the 2000x-diluted UC pellet with AntiSyntenin-555, Anti-CD63-647, Anti-CD9-488 and Anti-CD81-488. Internal cargo staining data are reported in Figure 3a. From this figure, it can be noted that there is a good correlation between capturing of the surface probe and internal cargo information that is specific for EVs. These data, together with the label-free sizing data and the colocalization results, confirm the small-EVs nature of the studied nanoparticles. Co-localization is high in this case, as well. Composite images that show co-localization are displayed in Figure $3 b$.

\subsection{Antibody density verification}

The probes for the binding kinetics IRIS experiments were spotted in a microarray modality, and an IRIS image of the chip prior to the experiment is reported in Figure 4a. As described in Section 2.5, to maintain a good spot conformation, the density of the active antibodies was tuned by keeping the same total concentration of the spotting solution (3 $\mathrm{mg} / \mathrm{mL}$ ) while varying the percentage of the active molecules. Therefore, even though the surface biomass density was maintained constant for all spots, resulting in similar intensities for all spots in Figure 4a, the percentage of active antibodies within each spot - ideally - should have changed linearly depending on the $\mathrm{IgG} 1 / \mathrm{IgG} 2$ ratio. However, since the yield of the immobilization process is not ideal, the actual amount of active probe within each spot was measured through a control experiment, by flowing a generic IgG1 antibody across the surface of a chip belonging to the same spotting batch as the one used for the EVs detection experiment. A differential image of the IRIS chip after the control experiment can be observed in Figure 4b.

The chip was first primed with buffer (PBS 1X) for 20 minutes, allowing for stabilization and acquisition of the baseline. Then, a generic IgG1 antibody was flowed across the surface for $20 \mathrm{~min}$ utes, at a flow speed of $200 \mathrm{uL} / \mathrm{min}$. A subsequent wash with buffer was performed to allow for the dissociation phase. The binding curves obtained through this experiment are displayed in figure 5. The curves were fitted with a bivalent model.

To derive the actual surface concentration of each antibody, we considered both the ratios of the initial slope and of the equilibrium points of the binding curves with those of the $100 \%$ spot. Those analysis are displayed in Figure 6. As expected, the data obtained from these two analyses are in good correlation, which implies that both could be used for an estimate of the active antibody concentration.

It can be noticed that, while the actual concentration of aCD81 in the spots is pretty consistent with the spotted concentration (The correlation in Fig. 6 is almost linear), the concentration of aCD63 and aCD9 seems to saturate with the spotted concentration. This is probably related to a lower immobilization yield of the antibodies or to 
the particular conformation of the spots. Nonetheless, we were able to retrieve the information of the actual concentration of the active antibody spots, necessary for the EVs capture experiment. Since the chips belong to the same spotting batch, they are indeed identical in terms of spotting conditions.

\subsection{Reflectance simulations}

With the IRIS technique, the accumulation of biomass on the sensor surface changes the optical path length, which results in a change in the measured reflectance signal. The reflectance from the IRIS substrate was simulated to understand the change in reflectance signal that results due to the accumulation of EVs on the sensor surface.

Figure 7 shows the change in reflectance signal in terms of the total reflectance $(\Delta R(d) / R)$ for a range of biomass accumulation (d) on an IRIS substrate with $110 \mathrm{~nm}$ of oxide. For this oxide thickness, the maximum change in reflectance signal is achieved using blue illumination $(\lambda=452 \mathrm{~nm})$ for small amounts of biomass accumulation (0-20nm). Since the small EVs are 50 to $150 \mathrm{~nm}$ in diameter, their accumulation on the surface surpasses the thickness where blue is optimal. Instead, green illumination $(\lambda=518 \mathrm{~nm})$ shows a greater change in reflectance for this biomass accumulation range, and was therefore chosen for these experiments.

\subsection{Real-time specific capture and de- tection of EVs}

The real-time characterization of an EVs sample was performed on the IRIS detection system by imaging different capture probe conditions simultaneously through the EVs solution, in order to optimize incubation conditions. A graphical representation of the experiment is presented in Figure 8.

As mentioned above, the chips used for these experiments belong to the same spotting batch of those used for the control experiments, and are therefore assumed to have the same concentration of active probes on the surface. The chip was primed with 1X PBS for 20 minutes prior to injection of EVs. The EVs sample was injected and recirculated for 20 minutes at a flow speed of $200 \mu \mathrm{L} / \mathrm{min}$. Images of the spots were acquired during the binding process, and during the following buffer wash that was performed to operate the dissociation phase.

As is also confirmed by Single-Particle ExoView measurements (Section 3.1), aCD81 has a higher affinity for capturing EVs with respect to aCD63 and aCD9, and is therefore the most efficient capture probe.

Considering the dissociation phase of the curves in Figure 9, one would notice that the slope is almost flat, meaning that the vesicles are well anchored to the surface. This is expected, assuming that each vesicle possesses on its surface multiple receptors, that will therefore bind to multiple antibody sites. The appearance of the binding data, and also the considerations stated above, clearly preclude the possibility of fitting the binding curves with a 1:1 Langmuir model. A multivalent model must be used, with an unknown number of binding sites to be considered per vesicle. We referred to the work by Li et al. [17] to derive a good model for the analysis of the binding curves of the exosomes to the surface probes. A more detailed discussion about the details of this fitting procedure are given in Section 4.2.

\section{Discussion}

\subsection{Incubation time optimization: in- flow dynamic capture}

Time optimization of EVs experiments is tricky: most EVs characterization methods involve $12 \mathrm{~h}$ or overnight incubations with dyes and other probes, both for capture and, successively, staining. Maximizing the binding efficiency is a priority, and that requires time, especially if the incubations are carried out in a static condition.

An incubation process is defined as static when no flow is involved; instead, a small quantity of sample is deposited in form of a droplet on the 
sensor, and the incubation is performed in a small humid chamber, which prevents the droplet from drying out. Obviously, this process only relies on diffusion mechanisms for the vesicles to explore the possible binding configurations. This method is widely used in DNA or protein fluorescence microarrays, and it works perfectly, since normally the binding agent is a molecule, and for most molecules brownian motion inside a droplet is enough to saturate all the binding sites in a very short time. However, EVs belong to the category of nanoparticles, and therefore, the brownian motion they experience is significantly lower than what molecules would in the same conditions. This implies that it takes a longer time for the particles to diffuse to the surface, explore the possible binding configurations, and, eventually, bind.

Here, we demonstrate that a constant speed flow allows the particles to bind fast and efficiently: in a 20 minute experiment, a high binding rate is achieved, especially on aCD81 spots. This result can be simply explained by considering how the flow helps the particles explore the available binding configurations in a more efficient way, maximizing their chance of finding a stable configuration on the surface. Moreover, even for high affinity binders, in our system, a flow rate of $200 \mu \mathrm{L} / \mathrm{min}$ is enough to minimize mass transport limitations [20]. Therefore, we demonstrate that a controlled flow system would improve the binding efficiency, and therefore reduce the incubation time, for any EVs related experiment.

\subsection{Probe density optimization: avid- ity effects}

Avidity effects occur when a nanoparticle that expresses more than one receptor molecules on its surface is captured on a surface that has been functionalized with a specific capture probe. Because of the multivalent nature of the reaction, the binding of the particle to the capture surface results in the occupation of more than one probe sites, therefore limiting the possibility for other particles to bind in its vicinity. Avidity therefore describes a mechanism of multivalent binding of multiple ligands to multiple probes and - depending on its range - it can improve or diminish the binding efficiency of the nanoparticles.

To study avidity effects in our system, we considered binding of EVs to different concentrations of the active probes. We considered the equilibrium binding signal from the EVs sample against the antibody surface density and we found (Figure 10) a good linear correlation, suggesting that the avidity regime does not change in this probe concentration range.

However, the avidity of a nanoparticle also characterizes the binding behavior and the shape of the binding curve. Li et al. [17] characterize the binding characteristics of nanoparticles by considering a percentage of fast binders and slow binders. Fast binders are defined as the particles that first reach the surface, when all the binding sites are completely available, and can therefore quickly find a configuration that allows them to bind in a fast, stable way, by occupying as many binding sites as possible. We define the binding rate of fast binders as $k_{O N, \text { fast }}$. On the other hand, slow binders are defined as the particles that reach the sensor surface at a later time, when more than half of the available binding sites are already occupied, and therefore, have a harder time finding a configuration that allows them to stably bind. When they bind, they will do so at a lower rate $\left(k_{O N, \text { slow }}\right)$ and each particle will occupy a lower number of binding sites with respect to a fast binder. Obviously, during the dissociation phase, fast binders - which are, by definition, bound in a more stable way will be less prone to leave the surface with respect to slow binders, which instead occupy a smaller number of binding sites. Therefore, in this case, the slow binders will dissociate with a higher rate, while the fast binders will dissociate with a lower rate.

To apply this model to fit the binding curves, we simulated binding curves by considering different percentages of fast binders and slow binders in the sample, each of them having its own set of association and dissociation constants, for a total of five different parameters $\left(k_{O N, \text { fast }}, k_{O F F, \text { fast }}, k_{O N, \text { slow }}\right.$, 
$\left.k_{O F F, \text { slow }}, \rho_{\text {fast } 2 \text { slow }}\right)$. The parameter $\rho_{\text {fast } 2 \text { slow }}$ is defined as the percentage of fast binders in the sample, and in our simulations we varied it from 0 to $100 \%\left(\rho_{\text {fast } 2 \text { slow }}=0\right.$ (no fast binders) to $\rho_{\text {fast } 2 \text { slow }}$ $=1$ (all fast binders)). The result of the simulations is displayed in Figure 11a. Here, we chose to vary the ratio of fast to slow binders because the amount of fast binders in the sample depends on the affinity of the probes, the size of the particles, the number of multivalent sites on each particle and the concentration of the sample. We then utilized the results of the simulations to decide which ratio of fast to slow binders was the most appropriate to fit our binding curves. The fitted binding curves for EVs binding to aCD9 and aCD81 spots are displayed on Figure $11 \mathrm{~b}$. Here, we chose a ratio of $\rho_{\text {fast } 2 s l o w}$ $=0.4$, meaning $40 \%$ of fast binders. Clearly, the model works well for this case, and the fitted data have a $R^{2}=0.998$. The obtained binding constants are in the order of the ones utilized for the simulations, which are: $k_{O N, \text { fast }}=10^{4} M^{-1} s^{-1}$, $k_{O F F, \text { fast }}=10^{-5} s^{-1}, k_{O N, \text { slow }}=10^{3} M^{-1} s^{-1}$, $k_{O F F, \text { slow }}=10^{-10} \mathrm{~s}^{-1}$.

\section{Conclusions}

We successfully demonstrate a label-free, multiplexed method for the optimization of the experimental parameters related to EVs experiments. This method would potentially allow researchers in the field of EVs characterization to dramatically reduce incubation time for EVs experiments, as well as optimize surface probe density, type of probe and other experimental parameters. Simul- taneous acquisition of binding of EVs to many different probes at different concentrations allowed for affinity characterization of the antibodies. A simplified multivalent model was used to analyze and fit binding curves of EVs, as detailed in Section 4.2. As expected, high affinity of EVs to aCD81 was demonstrated, and also confirmed by Single Particle Interferometric Imaging measurements.

\section{Acknowledgements}

This work was partially funded by the Boston University Ignition Program, by the National Science Foundation (NSF iCorps Award n'2027109 and NSF-TT PFI Award n¹941195), and by European Union's Horizon 2020 research and innovation program under grant agreement $\mathrm{n}^{\circ} 766466$ (INDEX).

\section{Declaration of Interest State- ment}

M.S. Ünlü is the principal investigator of the technology translation grants. He is the founder of a start up company (iRiS Kinetics, Inc.) for the commercialization of the multiplexed affinity technique.

\section{Supplementary material}

A supplementary PDF file with a scheme of the IRIS setup is available free of charge. 


\section{References}

[1] J. Karttunen, M. Heiskanen, A. Lipponen, D. Poulsen, and A. Pitkänen, "Extracellular vesicles as diagnostics and therapeutics for structural epilepsies," International journal of molecular sciences 20, p. 1259, 032019.

[2] G. van Niel, G. D'Angelo, and G. Raposo, "Shedding light on the cell biology of extracellular vesicles," Nature Reviews Molecular Cell Biology 19, pp. 213-228, Jan 2018.

[3] D. Raghu, J. A. Christodoulides, M. Christophersen, J. L. Liu, G. P. Anderson, M. Robitaille, J. M. Byers, and M. P. Raphael, "Nanoplasmonic pillars engineered for single exosome detection," PLOS ONE 13, pp. 1-13, 082018.

[4] R. van der Meel, M. Krawczyk-Durka, W. W. van Solinge, and R. M. Schiffelers, "Toward routine detection of extracellular vesicles in clinical samples," International Journal of Laboratory Hematology 36(3), pp. 244-253, 2014.

[5] T. Tian, Y.-L. Zhu, F.-H. Hu, Y.-Y. Wang, N.-P. Huang, and Z.-D. Xiao, "Dynamics of exosome internalization and trafficking," Journal of Cellular Physiology 228(7), pp. 1487-1495, 2013.

[6] X. Huang, R. O'Connor, and E. A. Kwizera, "Gold nanoparticle based platforms for circulating cancer marker detection," Nanotheranostics 1(1), pp. 80-102, 2017.

[7] O. Betzer, N. Perets, A. Angel, M. Motiei, T. Sadan, G. Yadid, D. Offen, and R. Popovtzer, "In vivo neuroimaging of exosomes using gold nanoparticles," ACS Nano 11, pp. 10883-10893, 11 2017.

[8] K. Liang, F. Liu, J. Fan, D. Sun, C. Liu, C. J. Lyon, D. W. Bernard, Y. Li, K. Yokoi, M. H. Katz, E. J. Koay, Z. Zhao, and Y. Hu, "Nanoplasmonic quantification of tumour-derived extracellular vesicles in plasma microsamples for diagnosis and treatment monitoring," Nature Biomedical Engineering 1(4), p. 0021, 2017.

[9] L. Grasso, R. Wyss, L. Weidenauer, A. Thampi, D. Demurtas, M. Prudent, N. Lion, and H. Vogel, "Molecular screening of cancer-derived exosomes by surface plasmon resonance spectroscopy," Analytical and Bioanalytical Chemistry 407, pp. 5425-5432, Apr 2015.

[10] Y. Fan, X. Duan, M. Zhao, X. Wei, J. Wu, W. Chen, P. Liu, W. Cheng, Q. Cheng, and S. Ding, "High-sensitive and multiplex biosensing assay of nsclc-derived exosomes via different recognition sites based on spri array," Biosensors and Bioelectronics 154, p. 112066, 2020.

[11] S. Picciolini, A. Gualerzi, R. Vanna, A. Sguassero, F. Gramatica, M. Bedoni, M. Masserini, and C. Morasso, "Detection and characterization of different brain-derived subpopulations of plasma exosomes by surface plasmon resonance imaging," Analytical Chemistry 90, pp. 8873-8880, Jul 2018.

[12] L. Zhu, K. Wang, J. Cui, H. Liu, X. Bu, H. Ma, W. Wang, H. Gong, C. Lausted, L. Hood, G. Yang, and $\mathrm{Z}$. Hu, "Label-free quantitative detection of tumor-derived exosomes through surface plasmon resonance imaging," Analytical Chemistry 86, pp. 8857-8864, 092014. 
[13] G. Daaboul, R. Vedula, S. Ahn, C. Lopez, A. Reddington, E. Ozkumur, and M. Ünlü, "Led-based interferometric reflectance imaging sensor for quantitative dynamic monitoring of biomolecular interactions," Biosensors and Bioelectronics 26(5), pp. 2221 - 2227, 2011.

[14] E. Chiodi, A. M. Marn, M. Geib, F. Ekiz Kanik, J. Rejman, D. AnKrapp, and M. Unlu, "Highly multiplexed label-free imaging sensor for accurate quantification of small-molecule binding kinetics," ACS Omega 5, pp. 25358-25364, 2020.

[15] C. A. Lopez, G. G. Daaboul, R. S. Vedula, E. Ozkumur, D. A. Bergstein, T. W. Geisbert, H. E. Fawcett, B. B. Goldberg, J. H. Connor, and M. S. Unlü, "Label-free multiplexed virus detection using spectral reflectance imaging," Biosensors \& bioelectronics 26, pp. 3432-3437, 042011.

[16] D. Sevenler and S. Unlu, "Numerical techniques for high-throughput reflectance interference biosensing," Journal of Modern Optics , 2015.

[17] M.-H. Li, S. K. Choi, P. R. Leroueil, and J. R. Baker, "Evaluating binding avidities of populations of heterogeneous multivalent ligand-functionalized nanoparticles," ACS Nano 8(6), pp. 5600-5609, 2014. PMID: 24810868.

[18] G. Pirri, F. Damin, M. Chiari, E. Bontempi, and L. E. Depero, "Characterization of a polymeric adsorbed coating for dna microarray glass slides," Analytical Chemistry 76(5), pp. 1352-1358, 2004. PMID: 14987092.

[19] C. Théry, K. W. Witwer, E. Aikawa, and M. J. A. et al., "Minimal information for studies of extracellular vesicles 2018 (misev2018): a position statement of the international society for extracellular vesicles and update of the misev2014 guidelines," Journal of Extracellular Vesicles 7(1), p. 1535750, 2018. PMID: 30637094.

[20] T. M. Squires, R. J. Messinger, and S. R. Manalis, "Making it stick: convection, reaction and diffusion in surface-based biosensors," Nature Biotechnology 26(4), pp. 417-426, 2008. 


\section{Figures}

a)

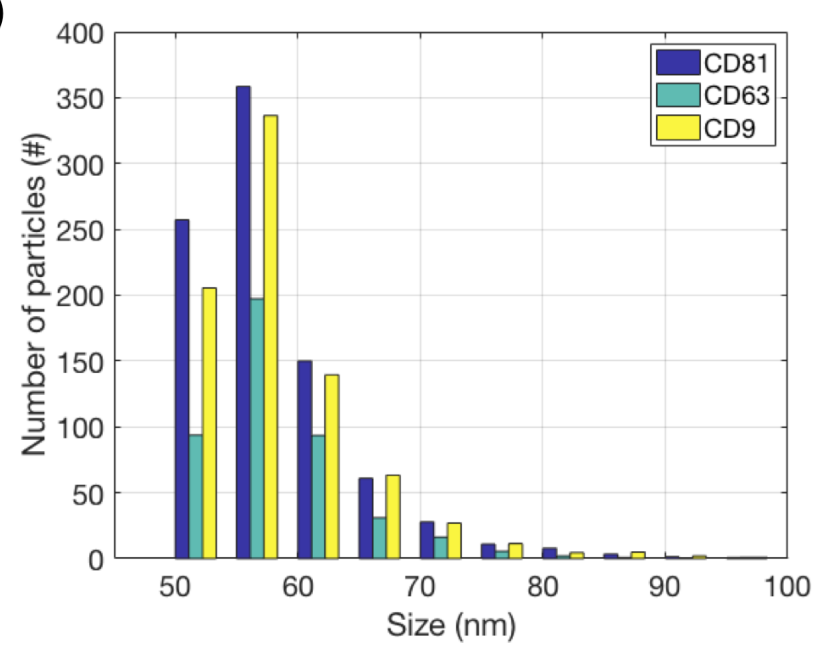

b)
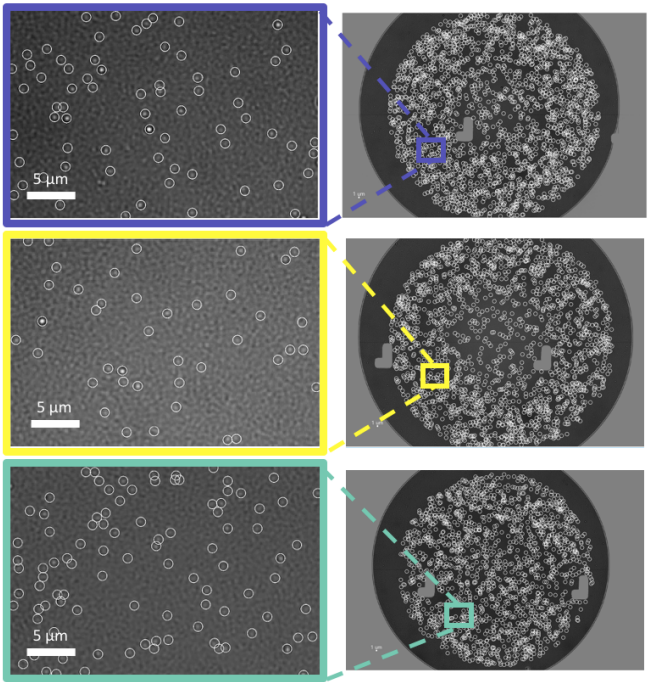

Figure 1: a) The size distribution of EVs captured on CD9, CD81, CD63 spots and b) label-free images of the particles on one of the spots.

a)

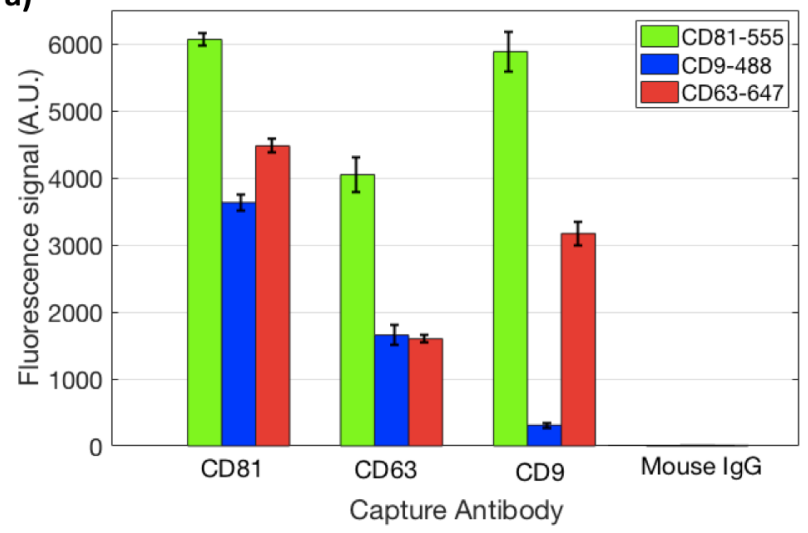

b)

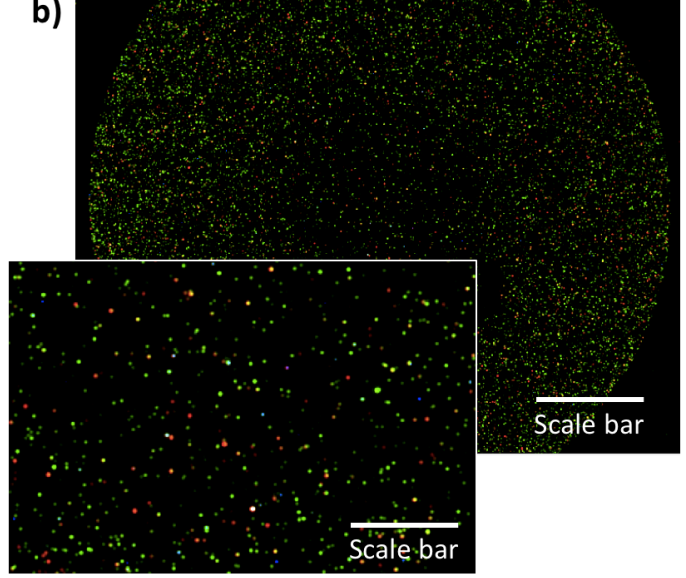

Figure 2: a) The fluorescence signal obtained for the analyzed EVs from staining with three different labeled antibodies (aCD81-555, aCD9-488, aCD63-647), on three different capture spots (aCD9, aCD81, aCD63). b) A co-localized fluorescence image of EVs captured on immobilized aCD9. Green points refer to CD81 staining, blue to CD9, red to CD63. The inset shows a zoomed-in image of the same spot. 
a)

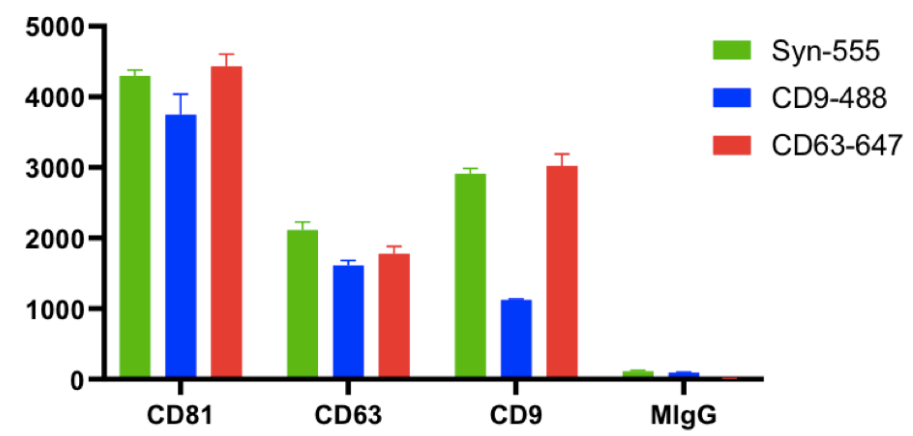

b)

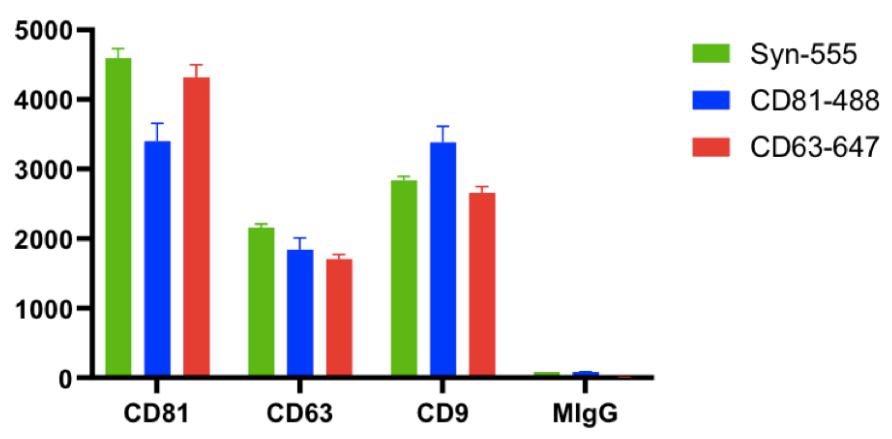

c)

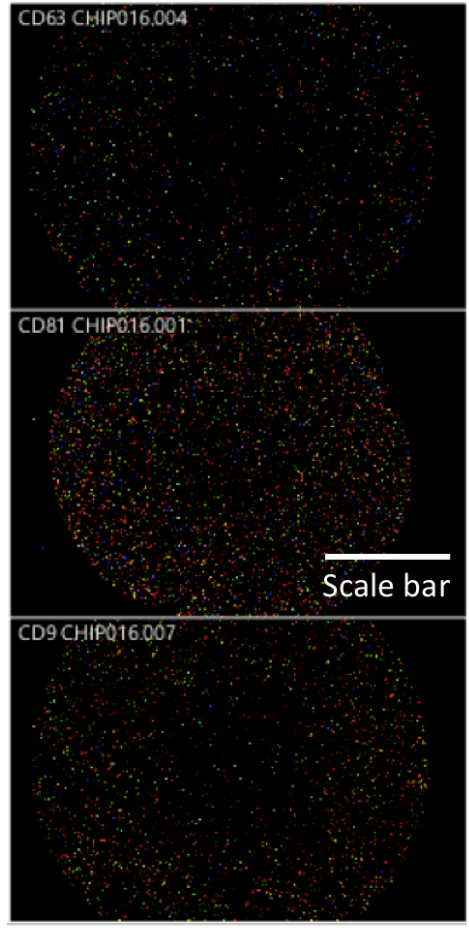

Figure 3: The fluorescence signal obtained for the analyzed EVs from staining the cargo with four different antibodies: a) Syn-555, CD9-488, CD63-647 and b) CD81-488, as they are captured on three different probe spots (aCD9, aCD63, aCD81). c) A co-localized fluorescence image of the three different capture spots on one of the chips used for the test.

a)

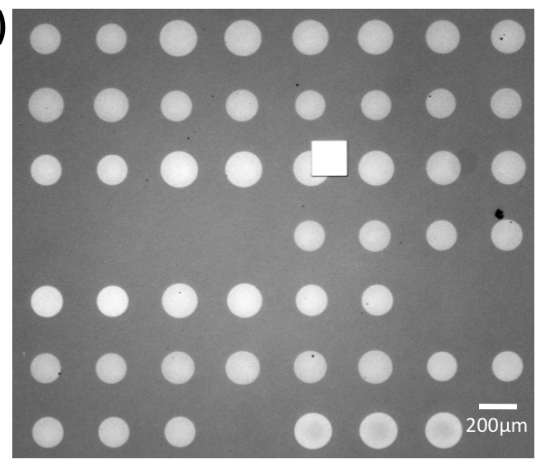

b)

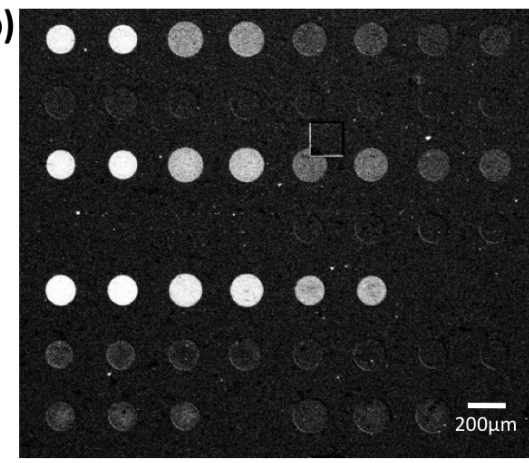

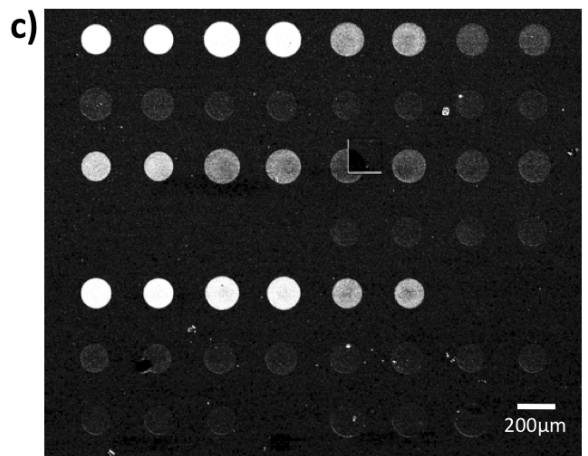

Figure 4: IRIS images of the chips utilized for the experiment a) before incubation, and differential images after incubation with b) antibodies and c) exosomes. To be noted that a similar level of signal doesn't necessarily correspond to the same increase in thickness, since the antibody experiment was carried out with blue LED illumination $(452 \mathrm{~nm})$ while the exosome capture was performed with green LED illumination $(512 \mathrm{~nm})$, to ensure a linear correlation between signal and thickness. 

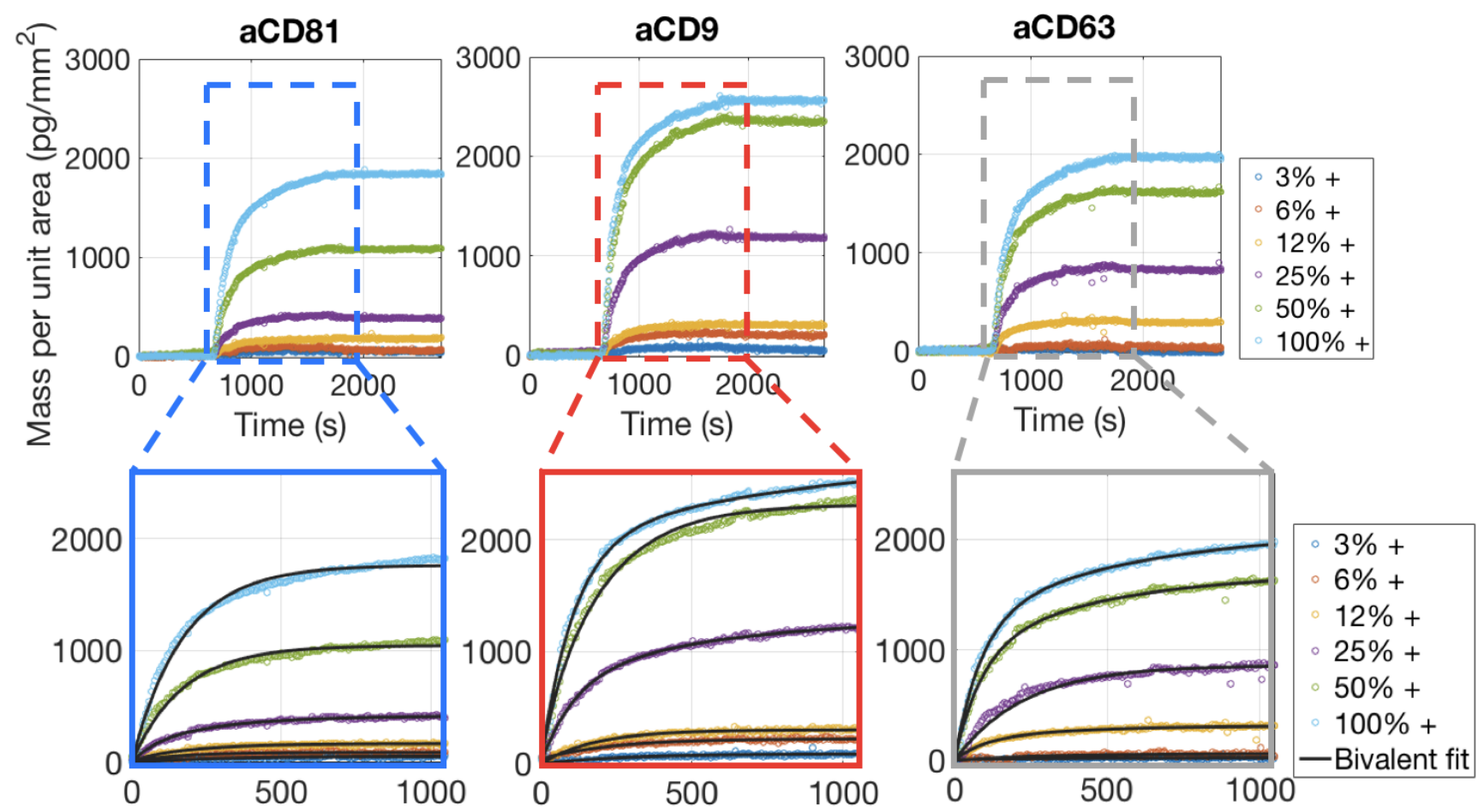

Figure 5: Binding curves of a generic IgG1 to one of the antibody chips used for the experiments. The insets focus on the association phase of the curves, which are fitted with a bivalent model.

Binding rate (M-IgG1 @5ug/mL)

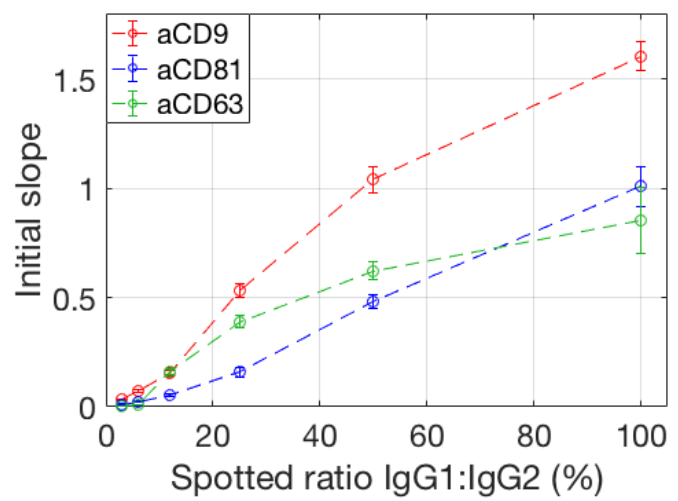

\section{Equilibrium binding signal (M-lgG1 @5ug/mL)}

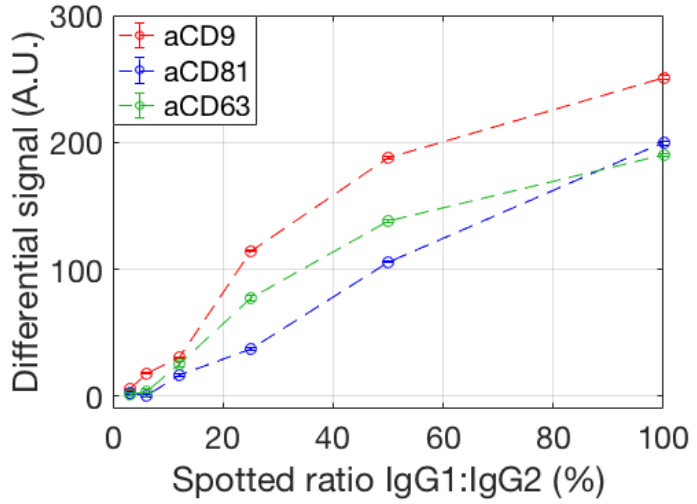

Figure 6: Verification of the amount of active antibody on the surface. The spotted percentage of active probe versus a) the initial slope of the binding curves in Figure 5 and b) the maximum signal obtained on the same dataset. 


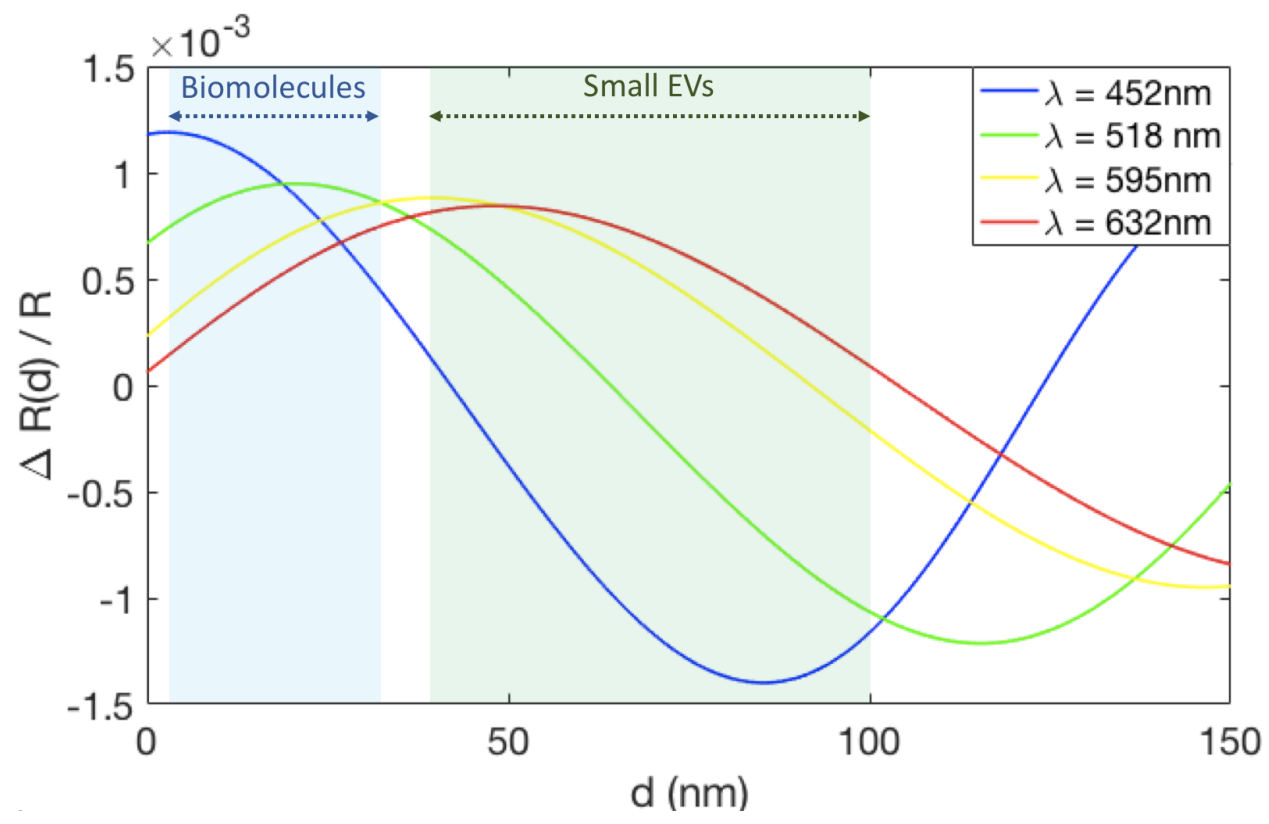

Figure 7: The change in reflectance signal due to biomass accumulation on an IRIS substrate with $110 \mathrm{~nm}$ of oxide for four different wavelengths, corresponding to the LEDs available on the IRIS system. The light blue region indicates the expected thicnkess range for biomolecules accumulation $(1-30 \mathrm{~nm})$, while the light green region shows the range expected for EVs $(40-100 \mathrm{~nm})$.

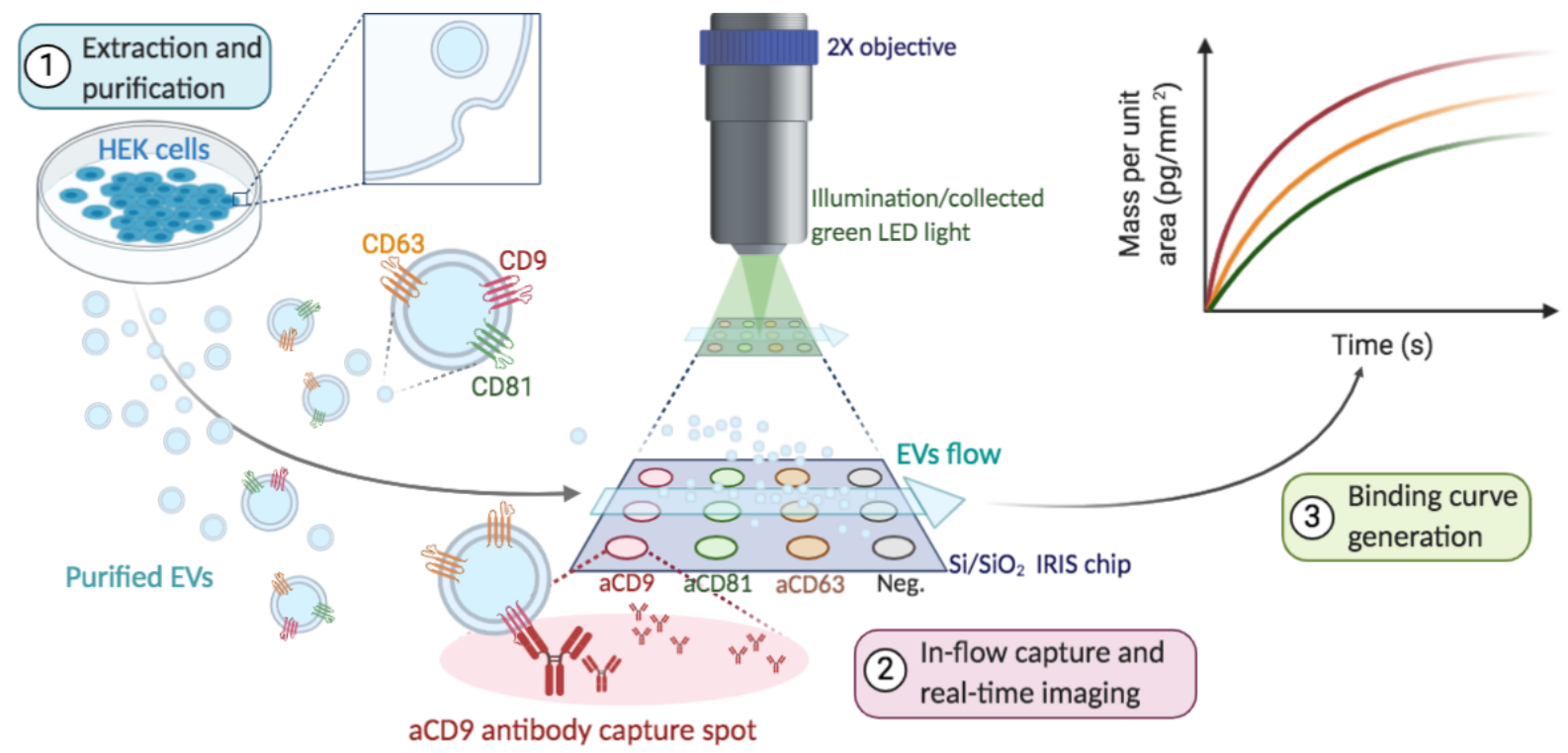

Figure 8: A scheme of the EVs detection experiment, from purification to detection and binding curve generation. Extracellular vesicles (EVs) are purified from HEK293T cell line by ultracentrifugation. The purified sample is diluted $2 \mathrm{X}$ and flowed across the surface of an IRIS chip where anti-tetraspanins were previously spotted in a microarray modality. Simultaneously, the reflectance from the surface is acquired, and the signal can be directly correlated to the thickness increase due to the accumulation of EVs. Finally, binding curves are generated. 


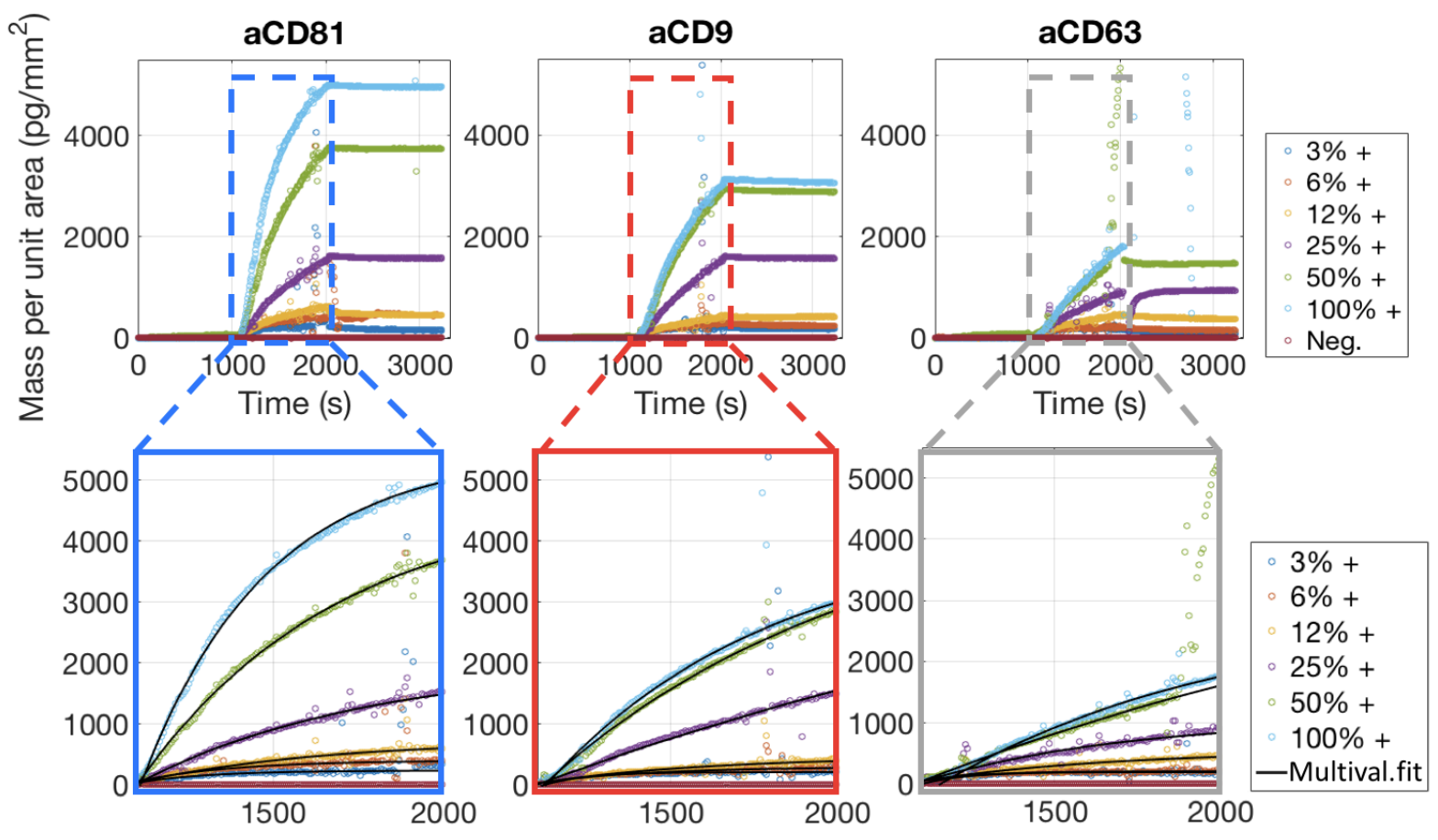

Figure 9: Simultaneously acquired real-time binding curves of EVs accumulating onto three different probes, each at six different concentrations. The insets focus on the association phase of the curves, which was fitted with a multivalent model that separates an initial, fast association rate $k_{o n, f a s t}$ from a slower, subsequent rate $k_{\text {on, slow }}$ as explained in the Discussion Section 4.2.

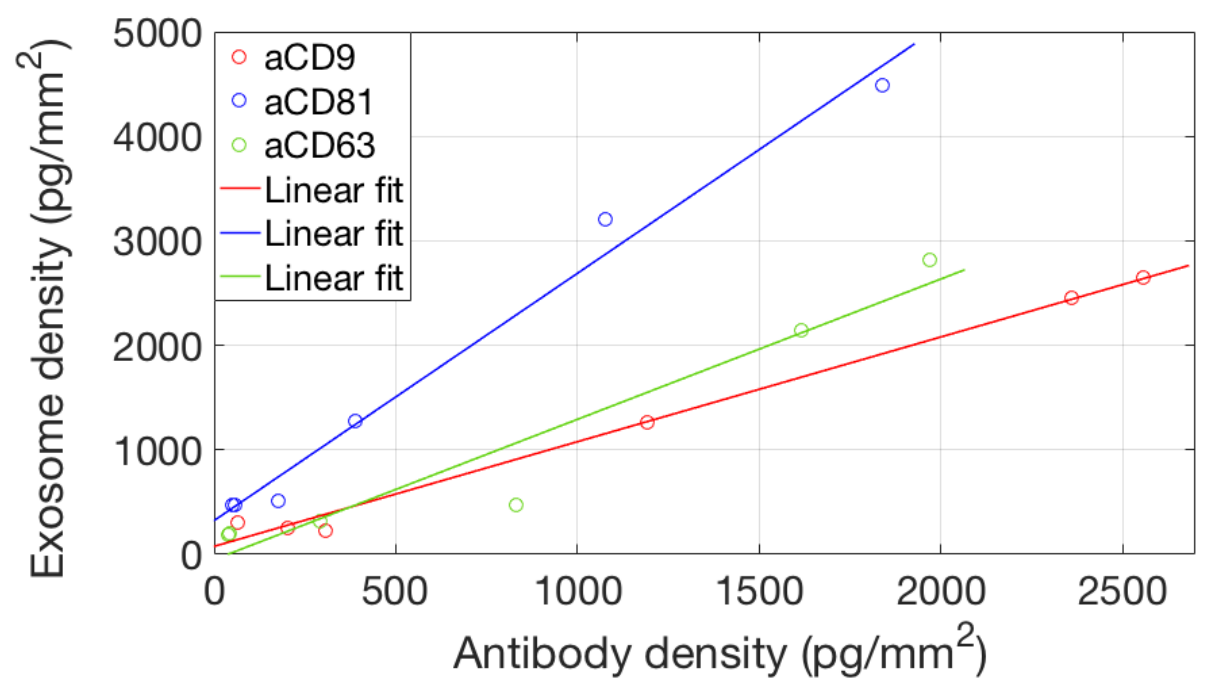

Figure 10: Correlation between the amount of active antibody immobilized on the surface and the total amount of captured EVs. The x coordinate of the data points corresponds to the maximum binding signal reached in Figure 5, while the y coordinate is the maximum EVs binding signal as shown in Figure 9. A linear correlation of the two datasets (no saturation) implies that - for this concentration ranges - the avidity regime does not change. 
a)

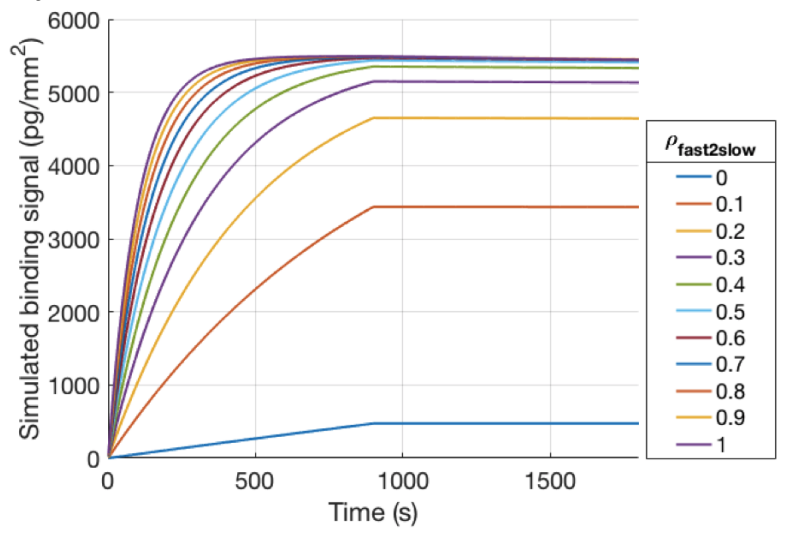

b)

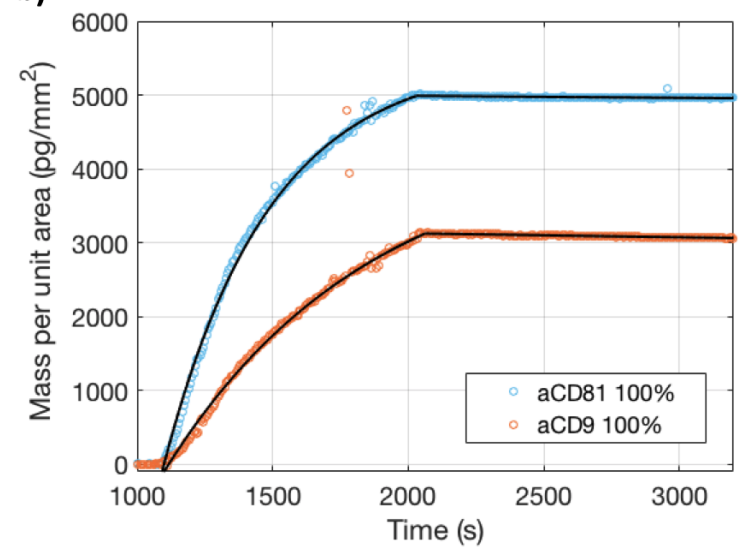

Figure 11: Comparison of simulated and real binding curves of EVs. a) Simulated curves, where increasing percentages of fast binders versus slow binders are considered. Here, $k_{O N \text {, fast }}=10^{4} M^{-1} \mathrm{~s}^{-1}$, $k_{O F F, \text { fast }}=10^{-5} s^{-1}, k_{O N, \text { slow }}=10^{3} M^{-1} s^{-1}, k_{O F F, \text { slow }}=10^{-10} s^{-1}$ at different percentage of fast and slow binders. model utilized for fitting EVs binding curves. 